\title{
Bifurcations and Symmetries of Optimal Solutions for Distributed Robotic Systems
}

\author{
Baoyang Deng, Mihir Sen and Bill Goodwine
}

\begin{abstract}
This paper studies bifurcations and multiple solutions of the optimal control problem for mobile robotic systems. While the existence of multiple local solutions to an optimization problem is not unexpected, the nature of the solutions are such that a relatively rich and interesting structure is present, which potentially could be exploited for controls purposes. In particular, this paper studies a group of unicyclelike autonomous mobile robots operating in a 2-dimensional obstacle-free environment. Each robot has a predefined initial state and final state and the problem is to find the optimal path between two states for every robot. The path is optimized with respect to the control effort and the deviation from a desired formation. The bifurcation parameter is the relative weight given to penalizing the deviation from the desired formation versus control effort. Numerically it is shown that as this number varies, bifurcations of solutions are obtained. Additional theoretic results of this paper relate to the symmetric properties of these bifurcations and the number and existence of multiple solutions for large and small values of the bifurcation parameter. Understanding the existence and nature of multiple solutions for optimization problems of this type is also of practical importance due the the ubiquity of gradient-based optimization methods where the search method will typically converge to the nearest local optimum.
\end{abstract}

\section{INTRODUCTION}

Distributed systems with multiple agents have been the focus of many research efforts in recent years. The applications of distributed systems are everywhere: robotic underwater vehicles [1], satellite clustering [2], electric power system [3], search and rescue operations [4] etc.

The approaches to the multi-robotic formation control problem are many and varied. Roughly, they can be categorized into three groups: leader-follower methods [5]-[7], behavior-based methods [8]-[10] and virtual structure methods [11]-[13]. In the leader-follower methods, each robot has at least one designated leader. Leaders can be some robots in the group or virtual robots that represent pre-computed trajectories supplied by a higher level planner. The other robots are followers that try to maintain a specified relative configuration to their leaders. Behavior-based methods draw inspiration from biology. In nature, animals in a group can combine their sensors to avoid their predators and search sufficient food. The behavior of each robot is prescribed and the final control is derived by weighting the relative

B. Deng is with the Department of Aerospace and Mechanical Engineering, University of Notre Dame, Notre Dame, IN 46556, USA bdeng2 and. edu

M. Sen is with Faculty of of Aerospace and Mechanical Engineering, University of Notre Dame, Notre Dame, IN 46556, USA msen a nd . edu

B. Goodwine is with Faculty of of Aerospace and Mechanical Engineering, University of Notre Dame, Notre Dame, IN 46556, USA jgoodwinend.edu importance of each behavior. The virtual structure methods involve the maintenance of a geometric configuration during robot movement using the idea that points in space should maintain a fixed geometric relationship. If robots behaved in this way, they would be moving inside a virtual structure.

In this paper, the problem addressed is to control a formation of robots moving along an optimal path between an initial configuration and a final configuration. The path is optimized with respect to a combination of the control effort and the deviation from a desired formation. Using standard methods from optimization, since each robot has its own predefined initial state and final state, the procedure to find the optimal path is to solve a boundary value problem ("BVP") for a set of second order ordinary differential equations ("ODEs").

The existence of multiple nontrivial solutions of BVPs for nonlinear second order ODEs have been investigated by many authors. For example, for

$$
\begin{aligned}
x^{\prime \prime}+a(t) f(x) & =0 \\
x(0) & =0 \\
x(1) & =0,
\end{aligned}
$$

the properties of the solutions depend on the limiting behavior of the function $f(u)$. Erbe and Wang [14] studied the existence of positive solutions of the equation with linear boundary conditions. Also, if

$$
\begin{aligned}
f_{0} & =\lim _{s \rightarrow+0} \frac{f(s)}{s} \\
f_{\infty} & =\lim _{s \rightarrow+\infty} \frac{f(s)}{s},
\end{aligned}
$$

they showed the existence of at least one positive solution in two cases, superlinearity $\left(f_{0}=0, f_{\infty}=\infty\right)$ or sublinearity $\left(f_{0}=\infty, f_{\infty}=0\right)$. In [15], Erbe, Hu and Wang showed that there were at least two positive solutions in the case of superlinearity at one end (zero or infinity) and sublinearity at the other end. Naito and Tanaka [16] and Ma and Thompson [17] established precise condition concerning the behavior of the ratio $f(s) / s$ for the existence and nonexistence of solutions. Their main results were that the BVP had at least $k$ solutions if the ratio $f(s) / s$ crossed the $k$ eigenvalues of the associated eigenvalue problem. For a class of systems of second order ODEs, Marcos do Ó, Lorca and Ubilla [18] used the fixed-point theorem of cone expansion/compression type, the upper-lower solutions method and degree arguments to study the existence. nonexistence, and multiplicity of positive solutions of the BVP. 
This paper first presents numerical results illustrating bifurcations and multiple solutions of the BVP associated with the optimal control problem. Then, it presents a theoretical result relating to the existence of multiple solutions in the limiting cases of small and large values of the bifurcation parameter. Finally, it proves the existence of symmetric solutions which guarantees that for any solution, a corresponding symmetric solution exists. The practical benefit of this result is that if a solution is found numerically, the symmetric solution can be computed from that algebraically.

\section{PROBLEM STATEMENT}

We adopt a simplified version of the robotic unicycle as a prototypical model. The simple kinematics of this kind of robot are described by

$$
\begin{aligned}
& \dot{x}=u_{1} \\
& \dot{y}=u_{2} .
\end{aligned}
$$

The problem is to find the controls $u_{i_{1}}(t), u_{i_{2}}(t)$ for each robot $i$ which steer a formation of robots of this type from the start configuration to its goal configuration, while maintaining a rigid body formation at the beginning and end of the trajectory and minimizing the global performance index

$$
J=\int_{0}^{t_{f}} \sum_{i=1}^{n}\left(\left(u_{i_{1}}\right)^{2}+\left(u_{i_{2}}\right)^{2}\right)+\sum_{i=1}^{n-1} k\left(d_{i}-\bar{d}\right)^{2} d t
$$

subject to the robotic kinematic constraints in Equation 1, where $n>2$ is the number of robots, $d_{i}=\left(\left(x_{i}-x_{i+1}\right)^{2}+\right.$ $\left.\left(y_{i}-y_{i+1}\right)^{2}\right)^{1 / 2}$ is the Euclidean distance from $i$ th to $(i+1)$ th robots, $\bar{d}$ is the desired distance between two adjacent robots, and $k$ is a non-negative weighting constant. The cost function minimizes a combination of the control effort (first summation) and the deviation from a desired formation (second summation).

Applying Pontryagin's maximum principle to solve the optimal control problem, we obtain the optimal inputs

$$
\begin{aligned}
u_{i_{1}} & =\frac{1}{2} p_{i_{1}} \\
u_{i_{2}} & =\frac{1}{2} p_{i_{2}},
\end{aligned}
$$

and equations of motion

$$
\begin{aligned}
\dot{x}_{i} & =\frac{1}{2} p_{i_{1}} \\
\dot{y}_{i} & =\frac{1}{2} p_{i_{2}} \\
\dot{p}_{i_{1}} & =\frac{2 k\left(x_{i}-x_{i-1}\right)\left(d_{i-1}-\bar{d}\right)}{d_{i-1}}+\frac{2 k\left(x_{i}-x_{i+1}\right)\left(d_{i}-\bar{d}\right)}{d_{i}} \\
\dot{p}_{i_{2}} & =\frac{2 k\left(y_{i}-y_{i-1}\right)\left(d_{i-1}-\bar{d}\right)}{d_{i-1}}+\frac{2 k\left(y_{i}-y_{i+1}\right)\left(d_{i}-\bar{d}\right)}{d_{i}} .
\end{aligned}
$$

Because they correspond to the robots at the end of the formation, the last two equations in Equation 2 only have the second term when $i=1$ and they only have the first term when $i=n$.
The cases considered in this paper are limited to the boundary conditions

$$
\begin{aligned}
& x_{i}(0)=c+(i-1) \bar{d}, \\
& x_{i}(1)=0, \\
& y_{i}(0)=0, \\
& y_{i}(1)=c+(i-1) \bar{d},
\end{aligned}
$$

where $c$ is a constant. These boundary conditions correspond to an initial formation with the robots arranged along the $x$ axis starting with the first robot at at $x=c$ with a distance $\bar{d}$ between each robot and a final formation with the robots arranged along the $y$-axis starting with the first robot at $y=c$ with a distance of $\bar{d}$ between each robot. It is important to note that if the initial and final formations are not parallel, then straight-line trajectories satisfying the boundary conditions will not, in general, maintain the desired distance between the robots.

\section{BIFURCATION RESULTS}

For a distributed system containing $n$ robots, when the weighting constant $k$ is given, an optimal trajectory can be obtained numerically by solving the equations of motion given by Equation 2 using the shooting method (see [19]).

\section{A. Solutions for a five robot system}

The figure on the left in Figure 1 illustrates three different solutions that satisfy the equations of motion in Equation 2 and boundary conditions in Equation 3 for $k=24.5, c=6$ and $\bar{d}=2$ for a formation of five robots. Since the differences among these trajectories are difficult to distinguish on such a small graph, the figure on the right illustrates them for the third (middle) robot with the difference magnified by a factor of 10 .
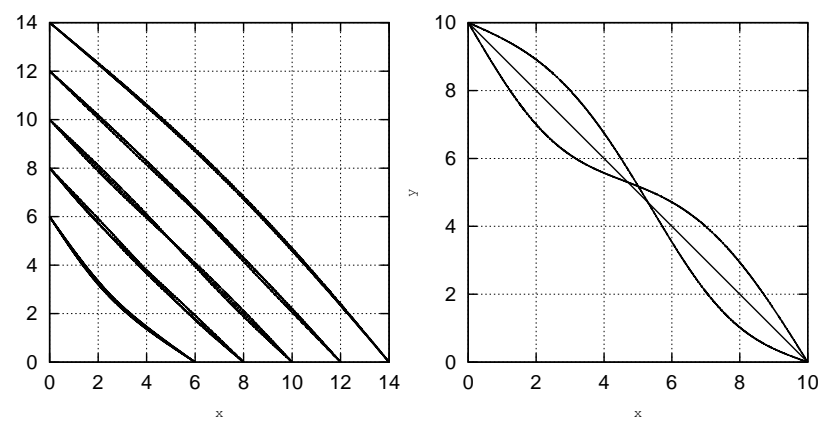

Fig. 1. Optimal paths for the five robot system with $k=24.5$.

Since $k$ is a parameter in differential equations, it will clearly affect the solutions. In fact, as $k$ is varied, the nature and number of solutions changes. Section IV shows that there is a unique solution when $k$ is small and in the limit as $k$ approaches infinity, the number of solutions also approaches infinity. In order to present the relationship between the number of solutions and $k$, we construct a bifurcation diagram as follows: since a straight line connecting end points is the optimal solution when $k=0$, we will designate that as a 
nominal trajectory. One measure of the difference between solutions would be their deviation from the straight line nominal solution at some specified time. As long as the different solution are not intersecting at that time, this would provide a measure of difference between different solutions. In all the bifurcation diagram illustrated subsequently, $t=$ 0.25 is used. For different formations and different type of robots, a different value of $t$ may be a better choice; however, for all the systems studied in this paper, $t=0.25$ appeared to adequately represent the relationship among the solutions. Also, alternative measures of differences between the solutions may, in general, be superior, this simple choice appears to suffice for all the cases considered in this paper.

The plots in Figure 2 illustrate this measure of the difference between solutions for each robot in the five robot system as $k$ is varied from 0 to 25 . In these bifurcation diagrams, the first robot is the one with the shortest trajectory, the fifth robot is the one with the longest trajectory and they are ordered sequentially. Observe that the bifurcation diagrams for robots 1 and 5 are symmetric to each other about $d=0$ axis and the bifurcation diagrams for robots 2 and 4 are similarly symmetric (even though each follows a trajectory with a different length). Finally, the bifurcation diagram for robot 3 is symmetric to itself about $d=0$ axis.

A close analysis of the actual trajectories that the robots follow illustrated in the figure on the right in Figure 1 reveals that the trajectories themselves are not symmetric (the two trajectories with pronounced curves intersect, but not at a point on the straight line solution). A measure that is based upon the deviation from the nominal solution appears to be necessary to determine the real symmetric nature of the solutions. Section V contains the analysis that these symmetries must, in fact, exist.

\section{B. Solutions for a seven robot system}

Figures 3 and 4 illustrate similar results for a seven robot system. Figure 3 illustrates the trajectories when $k=24.5$, $c=4$ and $\bar{d}=2$. Again, because the difference is hard to distinguish in the small left figure, the right figure in Figure 3 illustrates the trajectory with the deviation from the nominal trajectory for the fifth robot magnified by a factor of ten. Figure 4 illustrates the bifurcation diagrams for the solutions versus $k$ constructed in a manner identical to that of the system of five robots. Observe that, similar to the five robot case, the bifurcation diagrams for robots 1 and 7 are symmetric to each other about $d=0$ axis as is the bifurcation diagrams for robots 2 and 6 and robots 3 and 5, and the bifurcation diagram for robot 4 is symmetric to itself about $d=0$ axis.

\section{Asymptotic AnAlysis}

In the two cases of very small $k$ and very large $k$, we may use an asymptotic expansion to investigate the effect of $k$ on the number of solutions to the BVP. As will be shown, this analysis is consistent with the existence of a unique solution for small values of $k$ and many solutions for very large $k$, which is the pattern indicated in the numerical bifurcation
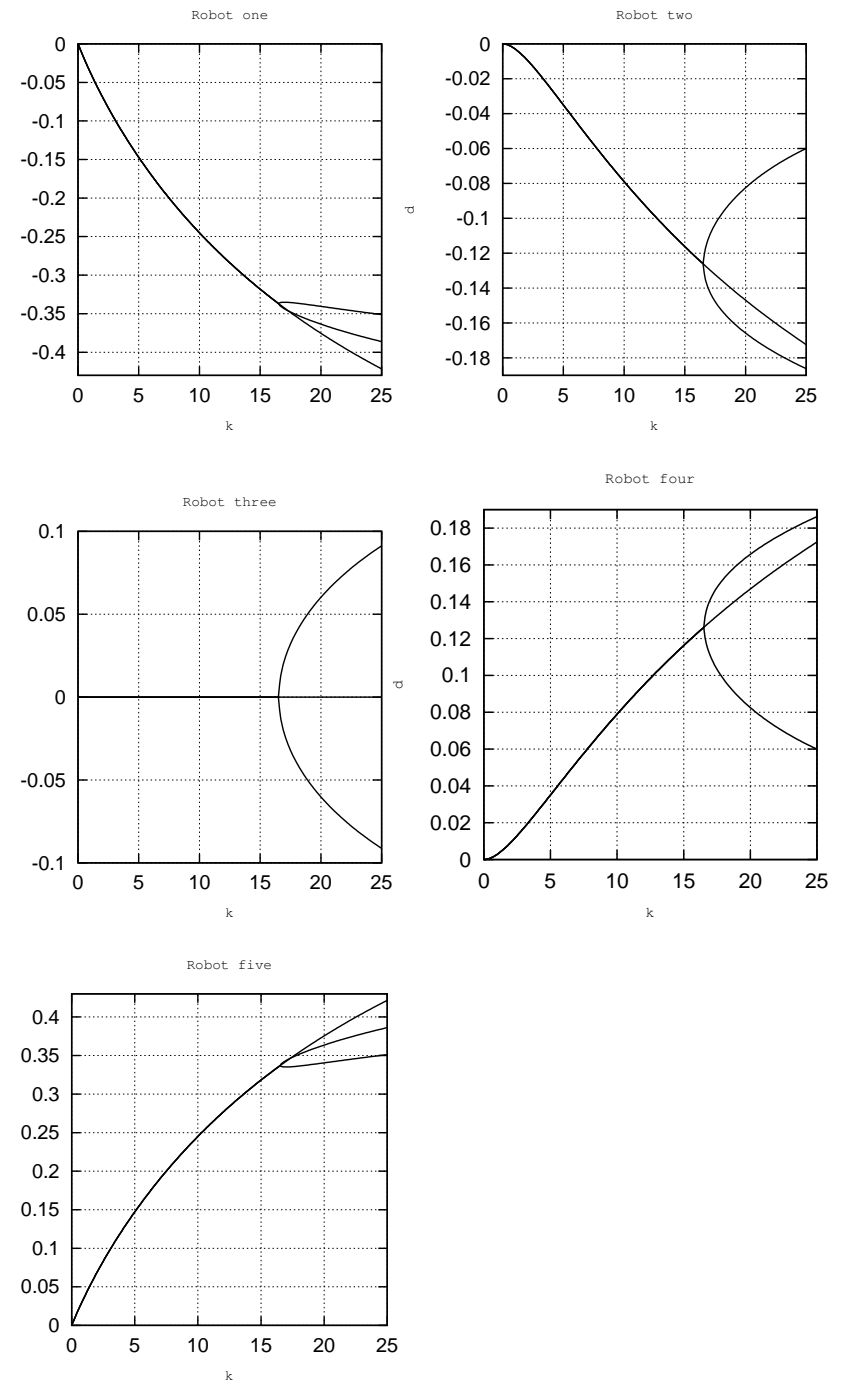

Fig. 2. Bifurcation diagrams for a five robot system.
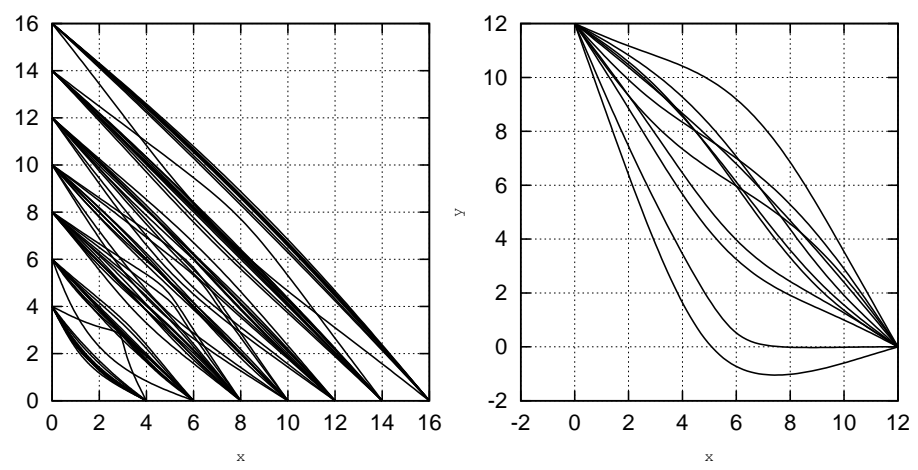

Fig. 3. Optimal paths for a seven robot system with $k=23$ 
results that show an increased number of bifurcations and an increased number of solutions as $k$ gets large.

\section{A. Small $k$}

We use a standard perturbation method (see [20]) to solve equations 2 for $k \ll 1$. If we let

$$
\begin{aligned}
x_{i} & =x_{i, 0}+k x_{i, 1}+k^{2} x_{i, 2}+k^{3} x_{i, 3}+\cdots+k^{j} x_{i, j}+\cdots, \\
y_{i} & =y_{i, 0}+k y_{i, 1}+k^{2} y_{i, 2}+k^{3} y_{i, 3}+\cdots+k^{j} y_{i, j}+\cdots, \\
p_{i_{1}} & =p_{i_{1}, 0}+k p_{i_{1}, 1}+k^{2} p_{i_{1}, 2}+k^{3} p_{i_{1}, 3}+\cdots+k_{j} p_{i_{1}, j}+\cdots, \\
p_{i_{2}} & =p_{i_{2}, 0}+k p_{i_{2}, 1}+k^{2} p_{i_{2}, 2}+k^{3} p_{i_{2}, 3}+\cdots+k_{j} p_{i_{2}, j}+\cdots
\end{aligned}
$$

and substitute into the equations of motion (Equation 2), a set of linear differential equations is obtained for each power of the expansion parameter $k$. Space limitations prevent the inclusion of the entire resulting equation, but we can consider it term-by-term in powers of $k$.

Specifically, if $z$ represents either $x$ or $y$, then the following table illustrates the resulting recursive structure of the equations. Any entry that is zero corresponds to a variable that is identically zero. Furthermore, as is the typical case in an asymptotic expansion, any variable only depends on lower order ones, which in this table correspond to variables to the left of it. Specifically, we have

\begin{tabular}{|c|cccccc|}
\hline$z_{i, 0}$ & $z_{i, 1}$ & $z_{i, 2}$ & $\cdots$ & $z_{i, m-1}$ & $z_{i, m}$ & $\cdots$ \\
\hline$z_{1,0}$ & $z_{1,1}$ & $z_{1,2}$ & $\cdots$ & $z_{1, m-1}$ & $z_{1, m}$ & $\cdots$ \\
$z_{2,0}$ & 0 & $z_{2,2}$ & $\cdots$ & $z_{2, m-1}$ & $z_{2, m}$ & $\cdots$ \\
$z_{3,0}$ & 0 & 0 & $\cdots$ & $z_{3, m-1}$ & $z_{3, m}$ & $\cdots$ \\
$\vdots$ & $\vdots$ & $\vdots$ & $\ddots$ & $\vdots$ & $\vdots$ & $\cdots$ \\
$z_{m, 0}$ & 0 & 0 & $\cdots$ & 0 & $z_{m, m}$ & $\cdots$ \\
$\vdots$ & $\vdots$ & $\vdots$ & $\ddots$ & $\vdots$ & $\vdots$ & $\cdots$ \\
$z_{n-2,0}$ & 0 & 0 & $\cdots$ & $-z_{3, m-1}$ & $-z_{3, m}$ & $\cdots$ \\
$z_{n-1,0}$ & 0 & $-z_{2,2}$ & $\cdots$ & $-z_{2, m-1}$ & $-z_{2, m}$ & $\cdots$ \\
$z_{n, 0}$ & $-z_{1,1}$ & $-z_{1,2}$ & $\cdots$ & $-z_{1, m-1}$ & $-z_{1, m}$ & $\cdots$ \\
\hline
\end{tabular}

where $m$ is the smallest integer larger than or equal to $\frac{n}{2}$. So, if $z_{i, i}$ is known and since $z_{i, j}(j>i)$ depends on $z_{i-1, j-2}, z_{i-1, j-1}, z_{i, j-2}, z_{i, j-1}, z_{i+1, j-2}, z_{i+1, j-1}$, we can solve them in the order of $j=i+1, i+2, \cdots$.

In detail, the $j=0\left(k^{0}\right)$ terms gives the set of linear equations

$$
\begin{aligned}
\dot{x}_{i, 0} & =\frac{1}{2} p_{i_{1}, 0} \\
\dot{y}_{i, 0} & =\frac{1}{2} p_{i_{2}, 0} \\
\dot{p}_{i_{1}, 0} & =0 \\
\dot{p}_{i_{2}, 0} & =0
\end{aligned}
$$

with boundary conditions

$$
\begin{aligned}
& x_{i 0}(0)=x_{10}(0)+(i-1) \bar{d} \\
& y_{i 0}(0)=0 \\
& x_{i 0}(1)=0 \\
& y_{i 0}(1)=y_{10}(1)+(i-1) \bar{d}
\end{aligned}
$$


which have solutions

$$
\begin{aligned}
x_{i, 0} & =-x_{i, 0}(0) t+x_{i, 0}(0), \\
y_{i, 0} & =y_{i, 0}(1) t \\
p_{i_{1}, 0} & =-2 x_{i, 0}(0) \\
p_{i_{2}, 0} & =2 y_{i, 0}(1) .
\end{aligned}
$$

Naturally, these are straight lines, which is expected when the only component of the cost function is the control effort and the 0th order solution does not contain $k$.

In all cases (all powers of $k$ and all robots), an analysis of the resulting expansion shows that $x_{i, j}=-x_{n+1-i, j}$ and $y_{i, j}=-y_{n+1-i, j}$. Also, for $1 \leq j<i \leq \frac{n+1}{2}, x_{i, j}=0$ and $y_{i, j}=$ 0 (the higher order terms for the "outer" robots are zero up to a certain order. Hence we only need to consider the cases where $1 \leq i \leq \frac{n}{2}$ and $i \leq j$.

In the case where $j=i=1$,

$$
\begin{aligned}
\dot{x}_{1,1} & =\frac{1}{2} p_{1_{1}, 1} \\
\dot{y}_{1,1} & =\frac{1}{2} p_{1_{1}, 2} \\
\dot{p}_{1_{1}, 1} & =2 \bar{d}(t-1)\left(1-\frac{1}{\sqrt{2 t^{2}-2 t+1}}\right) \\
\dot{p}_{1_{2}, 1} & =-2 \bar{d} t\left(1-\frac{1}{\sqrt{2 t^{2}-2 t+1}}\right) .
\end{aligned}
$$

Since the right hand sides of the last two equations are continuous and bounded functions of $t$ on the interval $\mathscr{I}=$ $[0,1]$, they are integrable and the integrals are differentiable (see [21]), which indicates the integrals are continuous. Hence $x_{1,1}, y_{1,1}$ exist and are unique since the right hand side of the $p$ equations may be directly integrated twice to obtain the $x$ and $y$ solutions. Since we integrate twice, there are two undetermined constants, which can be determined by the two zero boundary conditions.

When $i=j$ and $j>1$,

$$
\begin{aligned}
\dot{x}_{i, j} & =\frac{1}{2} p_{i_{1}, j} \\
\dot{y}_{i, j} & =\frac{1}{2} p_{i_{2}, j} \\
\dot{p}_{i_{1}, j} & =-2 x_{i-1, j-1}+\frac{2 t\left(-y_{i-1, j-1}+t\left(x_{i-1, j-1}+y_{i-1, j-1}\right)\right)}{\left(2 t^{2}-2 t+1\right)^{3 / 2}} \\
\dot{p}_{i_{2}, j} & =2 y_{i-1, j-1}+\frac{2(t-1)\left(-y_{i-1, j-1}+t\left(x_{i-1, j-1}+y_{i-1, j-1}\right)\right)}{\left(2 t^{2}-2 t+1\right)^{3 / 2}} .
\end{aligned}
$$

The right hand sides of the last two equations are the sum of integrable functions or product of them, so they are differentiable (see [21]). Similar to the argument for $x_{1,1}$ and $y_{1,1}, x_{i, i}$ and $y_{i, i}$ therefore exist and unique.

Space limitations prevent the detailed inclusion of the expressions for the off-diagonal terms. However, they have the same essential structure that the right hand side of the co-state equations is a linear combination of the lower order solutions in the expansion. Since all the lower order solutions are continuous and bounded functions of $t$, they may be directly integrated to compute the actual solution.
Since all the terms in the expansion may be solved by direction integration of functions that are continuous and bounded, a solution for each term exists. Hence, for $k \ll 1$, this asymptotic analysis give a computable construction for the solutions, and also indicates that the solution is unique. In other words, for small $k$, only one solution exists.

\section{B. Large $k$}

For large $k\left(\frac{1}{k} \ll 1\right)$, a similar asymptotic expansion is used to solve equations 2 but instead of $k, \varepsilon=\frac{1}{k}$ is used as the expansion parameter. Let

$$
\begin{aligned}
x_{i} & =x_{i, 0}+\varepsilon x_{i, 1}+\varepsilon^{2} x_{i, 2}+\varepsilon^{3} x_{i, 3}+\cdots+\varepsilon^{j} x_{i, j}+\cdots, \\
y_{i} & =y_{i, 0}+\varepsilon y_{i, 1}+\varepsilon^{2} y_{i, 2}+\varepsilon^{3} y_{i, 3}+\cdots+\varepsilon^{j} y_{i, j}+\cdots, \\
p_{i_{1}} & =p_{i_{1}, 0}+\varepsilon p_{i_{1}, 1}+\varepsilon^{2} p_{i_{1}, 2}+\varepsilon^{3} p_{i_{1}, 3}+\cdots+\varepsilon_{j} p_{i_{1}, j}+\cdots, \\
p_{i_{2}} & =p_{i_{2}, 0}+\varepsilon p_{i_{2}, 1}+\varepsilon^{2} p_{i_{2}, 2}+\varepsilon^{3} p_{i_{2}, 3}+\cdots+\varepsilon_{j} p_{i_{2}, j}+\cdots .
\end{aligned}
$$

We obtain the following equation for leading order of $\varepsilon$,

$$
\begin{aligned}
\dot{x}_{i, 0} & =\frac{1}{2} p_{i_{1}, 0} \\
\dot{y}_{i, 0} & =\frac{1}{2} p_{i_{2}, 0} \\
0 & =\frac{2 k\left(x_{i, 0}-x_{i-1,0}\right)\left(d_{i-1,0}-\bar{d}\right)}{d_{i-1,0}}+\frac{2 k\left(x_{i, 0}-x_{i+1,0}\right)\left(d_{i, 0}-\bar{d}\right)}{d_{i, 0}} \\
0 & =\frac{2 k\left(y_{i, 0}-y_{i-1,0}\right)\left(d_{i-1,0}-\bar{d}\right)}{d_{i-1,0}}+\frac{2 k\left(y_{i, 0}-y_{i+1,0}\right)\left(d_{i, 0}-\bar{d}\right)}{d_{i 0}} .
\end{aligned}
$$

The last two equations may be simplified to

$$
\left(x_{i, 0}-x_{i-1,0}\right)^{2}+\left(y_{i, 0}-y_{i-1,0}\right)^{2}=\bar{d}^{2},
$$

which transparently shows that the limit for large $k$ simply requires that the distance constraint be exactly maintained.

Since the third and fourth equations are algebraic (as is Equation 4), then the costates, $p$ are unconstrained and hence any path that maintains the desired distance between the robots and satisfies the boundary conditions is a solution. This makes intuitive sense: in the limit as $k \rightarrow \infty$, the control effort becomes negligible relative to the distance constraint. Hence, in the limit of very large $k$, the asymptotic analysis indicates that there is an infinite number of solutions. As long as the separation distance is maintained and the boundary conditions are satisfied, any path is optimal.

\section{Symmetries in The BifurCATION Diagrams}

This section proves that the symmetries found in the numerically-constructed bifurcation diagrams must be present. This is of practical value because it reduces the computation time necessary in a search over multiple solutions since a second solution can always be found from any solution that is obtained (unless the solution is symmetric with itself).

Suppose $\left(x_{1}, x_{2}, \cdots, x_{n}, y_{1}, y_{2}, \cdots, y_{n}\right)$ is a solution of Equation 2 with the boundary conditions in Equation 3, and let

$$
\begin{aligned}
& x_{i}=\left(x_{s}\right)_{i}+\left(x_{d}\right)_{i}, \\
& y_{i}=\left(y_{s}\right)_{i}+\left(y_{d}\right)_{i},
\end{aligned}
$$


where

Proposition 1: Suppose $v(t)$ is a fixed point of equation 7.

$$
\begin{aligned}
\left(x_{s}\right)_{i} & =(c+(i-1) \bar{d})(1-t), \\
\left(y_{s}\right)_{i} & =(c+(i-1) \bar{d}) t .
\end{aligned}
$$

The subscripts $s$ indicate a "straight-line" solution and the subscripts $d$ indicate the component of the solution that is a "deviation" from the straight line. If $v(t)=\left(\left(x_{d}\right)_{1},\left(y_{d}\right)_{1}, \cdots,\left(x_{d}\right)_{n},\left(y_{d}\right)_{n}\right)$, then $\left(x_{d}\right)_{i},\left(y_{d}\right)_{i}, \quad i=$ $1,2, \cdots, n$, satisfy the following equations with homogeneous boundary conditions:

$$
\begin{aligned}
& -\left(\ddot{x}_{d}\right)_{i}(t)=f_{i}(v(t)), \\
& -\left(\ddot{y}_{d}\right)_{i}(t)=g_{i}(v(t)),
\end{aligned}
$$

where $f_{1}=h_{1}, g_{1}=l_{1}, f_{n}=-h_{n-1}, g_{n}=-l_{n-1}$, and for $i=(2,3, \cdots, n-1)$

$$
\begin{aligned}
f_{i} & =h_{i}-h_{i-1}, \\
g_{i} & =l_{i}-l_{i-1}
\end{aligned}
$$

where, for all $i=(1,2, \cdots, n)$

$$
\begin{aligned}
h_{i} & =\left(\frac{\bar{d}}{d_{i}}-1\right)\left(-\bar{d}+\bar{d} t+\left(x_{d}\right)_{i}-\left(x_{d}\right)_{i+1}\right), \\
l_{i} & =\left(\frac{\bar{d}}{d_{i}}-1\right)\left(-\bar{d} t+\left(y_{d}\right)_{i}-\left(y_{d}\right)_{i+1}\right), \\
d_{i} & =\left(\left(-\bar{d}+\bar{d} t+\left(x_{d}\right)_{i}-\left(x_{d}\right)_{i+1}\right)^{2}+\left(-\bar{d} t+\left(y_{d}\right)_{i}-\left(y_{d}\right)_{i+1}\right)^{2}\right)^{\frac{1}{2}}
\end{aligned}
$$

The system (5), is equivalent to the system of integral equations

$$
\begin{aligned}
& \left(x_{d}\right)_{i}=\int_{0}^{1} G(t, s) f_{i}(v(s)) d s, \\
& \left(y_{d}\right)_{i}=\int_{0}^{1} G(t, s) g_{i}(v(s)) d s,
\end{aligned}
$$

where $G(t, s)$ is the Green's function of the differential operator $-\ddot{u}=0$ with homogeneous boundary conditions, where $u=x_{d_{i}}$ or $u=y_{d_{i}}$, and

$$
G(t, s)=\left\{\begin{array}{ll}
t(1-s), & t \leq s \\
s(1-t), & t>s
\end{array} .\right.
$$

If $A_{i}, B_{i}$ and $F$ are maps such that

$$
\begin{aligned}
A_{i} v(t) & =k \int_{0}^{1} G(t, s) f_{i}(v(s)) d s, \\
B_{i} v(t) & =k \int_{0}^{1} G(t, s) g_{i}(v(s)) d s, \\
F v(t) & =\left(A_{1}(v)(t), B_{1}(v)(t), \cdots, A_{n}(v)(t), B_{n}(v)(t),\right.
\end{aligned}
$$

then determining a solution to equation (6) is equivalent to finding a fixed point to equation

$$
F v(t)=v(t) .
$$

The following proposition proves that if a solution is known, then the "opposite" deviation from the straight-line solution is also a solution for the robot on the other side of the formation. Let

$$
\begin{aligned}
& \left(\hat{x}_{d}\right)_{n+1-i}=-\left(x_{d}\right)_{i} \\
& \left(\hat{y}_{d}\right)_{n+1-i}=-\left(y_{d}\right)_{i}
\end{aligned}
$$

and $\hat{v}(t)=\left(\left(\hat{x}_{d}\right)_{1},\left(\hat{y}_{d}\right)_{1}, \cdots,\left(\hat{x}_{d}\right)_{n},\left(\hat{y}_{d}\right)_{n}\right)$, then $\hat{v}(t)$ is also a fixed point of equation 7

Proof: The proof is by direct substitution. Substituting for the definition of the hat terms for each gives:

$$
\begin{aligned}
d_{i} & =\sqrt{\left(-\bar{d}+\bar{d} t+\left(x_{d}\right)_{i}-\left(x_{d}\right)_{i+1}\right)^{2}+\left(-\bar{d} t+\left(y_{d}\right)_{i}-\left(y_{d}\right)_{i+1}\right)^{2}} \\
& =\sqrt{\left(-\bar{d}+\bar{d} t-\left(\hat{x}_{d}\right)_{n+1-i}+\left(\hat{x}_{d}\right)_{n-i}\right)^{2}+\left(-\bar{d} t-\left(\hat{y}_{d}\right)_{n+1-i}+\left(\hat{y}_{d}\right)_{n-i}\right)^{2}} \\
& =\sqrt{\left(-\bar{d}+\bar{d} t+\left(\hat{x}_{d}\right)_{n-i}-\left(\hat{x}_{d}\right)_{n-i+1}\right)^{2}+\left(-\bar{d} t+\left(\hat{y}_{d}\right)_{n-i}-\left(\hat{y}_{d}\right)_{n-i+1}\right)^{2}} \\
& =\hat{d}_{n-i} \\
h_{i} & =\left(\frac{\bar{d}}{d_{i}}-1\right)\left(-\bar{d}+\bar{d} t+\left(x_{d}\right)_{i}-\left(x_{d}\right)_{i+1}\right) \\
& =\left(\frac{\bar{d}}{\hat{d}_{n-i}}-1\right)\left(-\bar{d}+\bar{d} t-\left(\hat{x}_{d}\right)_{n+1-i}+\left(\hat{x}_{d}\right)_{n-i}\right) \\
& =\left(\frac{\bar{d}}{\hat{d}_{n-i}}-1\right)\left(-\bar{d}+\bar{d} t+\left(\hat{x}_{d}\right)_{n-i}-\left(\hat{x}_{d}\right)_{n-i+1}\right) \\
& =\hat{h}_{n-i} \\
l_{i} & =\left(\frac{\bar{d}}{d_{i}}-1\right)\left(-\bar{d} t+\left(y_{d}\right)_{i}-\left(y_{d}\right)_{i+1}\right) \\
& =\left(\frac{\bar{d}}{\hat{d}_{n-i}}-1\right)\left(-\bar{d} t-\left(\hat{y}_{d}\right)_{n+1-i}+\left(\hat{y}_{d}\right)_{n-i}\right) \\
& =\left(\frac{\bar{d}}{\hat{d}_{n-i}}-1\right)\left(-\bar{d} t+\left(\hat{y}_{d}\right)_{n-i}-\left(\hat{y}_{d}\right)_{n-i+1}\right) \\
& =\hat{l}_{n-i}
\end{aligned}
$$

and

$$
\begin{aligned}
f_{1} & =h_{1}=\hat{h}_{n-1}=-\hat{f}_{n} \\
g_{1} & =l_{1}=\hat{l}_{n-1}=-\hat{g}_{n} \\
f_{i} & =h_{i}-h_{i-1}=\hat{h}_{n-i}-\hat{h}_{n+1-i}=-\hat{f}_{n+1-i} \\
g_{i} & =l_{i}-l_{i-1}=\hat{l}_{n-i}-\hat{h}_{n+1-i}=-\hat{g}_{n+1-i} \\
f_{n} & =-h_{n-1}=-\hat{h}_{1}=-\hat{f}_{1} \\
g_{n} & =-l_{n-1}=-\hat{l}_{1}=-\hat{g}_{1}
\end{aligned}
$$

which give us

$$
\begin{aligned}
& f_{i}=-\hat{f}_{n+1-i}, \\
& g_{i}=-\hat{g}_{n+1-i} .
\end{aligned}
$$

for all $i$ from 0 to $n$. Then

$$
\begin{gathered}
\left(\hat{x}_{d}\right)_{i}=-\left(x_{d}\right)_{n+1-i}=-\int_{0}^{1} G(t, s) f_{n+1-i} d s=\int_{0}^{1} G(t, s) \hat{f}_{i} d s \\
\left(\hat{y}_{d}\right)_{i}=-\left(y_{d}\right)_{n+1-i}=-\int_{0}^{1} G(t, s) g_{n+1-i} d s=\int_{0}^{1} G(t, s) \hat{g}_{i} d s .
\end{gathered}
$$

Hence $\left.\hat{v}(t)=\left(\hat{x}_{d}\right)_{1},\left(\hat{y}_{d}\right)_{1}, \cdots,\left(\hat{x}_{d}\right)_{n},\left(\hat{y}_{d}\right)_{n}\right)$ is a solution of equation 7 .

Equation 8 gives an algebraic expression for the symmetric solutions, which is useful because the theorem proves they satisfy the boundary value problems and hence reduces the computational burden of determining additional solutions. Note that the relationship is not simply the opposite deviation from the straight line solution, but is the opposite deviation from the straight line for a different robot. 


\section{CONCLUSIONS AND FUTURE WORKS}

\section{A. Conclusions}

This paper considers the optimal control problem for a formation of multiple robots. The trajectory of each robot is optimized with respect to a combination of the control effort and the deviation from a desired formation, which in this paper is simply a formation that maintains a specified distance between adjacent robots. The paper first presents numerical results illustrating the structure of bifurcations and multiple solutions of the BVP associated with the optimal control problem. Then it shows that an asymptotic analysis indicates that there is a unique solution when $k$ is small and in the limit as $k$ approaches infinity, the number of solutions also approaches infinity. Then, it presents a theoretical result relating to the existence of symmetric solutions. It guarantees that for any solution, a corresponding symmetric solution exists. The practical benefit is that if a solution found numerically, the symmetric solution can be computed from that algebraically. Also, if a gradient-based search method is used, understanding of the structure of the relationship among multiple solutions is necessary to find the desired resul.t Finding multiple solutions may be desirable if the cost function does not include all the optimization criteria; for example, if obstacles are present but not accounted for in the cost function.

\section{B. Future work}

Future work is directed in several areas. The results are likely to be much more general than the particular case presented in this paper. Determining the most general classes of robots and formations that maintain the symmetry properties of the results and similar bifurcation structure is of interest. Also, the asymptotic analysis is only of any use for the limiting values for $k$. Determining conditions for the existence of a bifurcation for any value of $k$, similar to that for initial value problems, would be useful.

\section{REFERENCES}

[1] T. R. Smith, H. Hanssmann, and N. E. Leonard, "Orientation control of multiple underwater vehicles with symmetry-breaking potentials," In 40th IEEE Conference on Decision and Control, pp. 4598-4603, 2001.

[2] C. R. McInnes, "Autonomous ring formation for a planar constellation of satellites," AIAA Journal of Guidance Control and Dynamics, vol. 18, no. 5, pp. 1215-1217, 1995.

[3] H. Puttgen, P. MacGrego, and F. Lambert, "Distributed generation: semantic hype or dawn of a new era," IEEE Power and Energy Magazine, vol. 1, pp. 22-29, 2003.

[4] J. Jennings, G. Whelan, and W. Evans, "Cooperative search and rescue with a team of mobile robots," in IEEE International Conference on Advanced Robotics, 1997, pp. 193-200.

[5] J. P. Desai, "Modeling multiple teams of mobile robots: a graph theoretic approach," in Proceedings of the 2001 IEEE/RSJ International Conference on Intelligent Robots ans Systems, 2001, pp. 381-386.

[6] J. P. Desai, J. Ostrowski, and V. Kumar, "Controlling formation of multiple mobile robots," in IEEE International Conference on Robotics and Automation, 1998, pp. 16-21.

[7] N. E. Leonard and E. Fiorelli, "Virtual leaders, artificial potentials, and coordinated control of groups," In 40th IEEE Conference on Decision and Control, pp. 2968-2973, 2001.

[8] T. Balch and R. Arkin, "Behavior-based formation control for mutirobotic teams," IEEE Transactions on Robotics and Automation, vol. 14, no. 6, pp. 926-934, 1998.
[9] T. Balch and M. Hybinette, "Behavior-based coordination of largescale robot formations," in Fourth International Conference on MultiAgent Systems, 2000.

[10] Z. Su and J. Lu, "Formation feedback applied to behavior-based approach to formation keeping," Jounal of Beijing Institute of Technology, vol. 13, no. 2, pp. 190-193, 2004.

[11] C. Belta and V. Kumar, Geometric Methods for Multi-Robot optimal Motion Planning, Handbook of Computational Geometry for Pattern Recognition, Computer Vision, Neurocomputing and Robotics, E. Bayro-Corrochano, Ed. Berlin: Springer-Verlag, 2005.

[12] M. A. Lewis and K. H. Tan, "High precision formation control of mobile robots using virtual structures," Autonomous Robots, vol. 4, pp. 387-403, 1997.

[13] K.-H. Tan and M. A. Lewis, "Virtual structures for high-precision cooperative mobile robotic control," International Conference on Intelligent Robots and Systems, pp. 132-139, 1996.

[14] L. Erbe and H. Wang, "On the existence of positive solutions of ordinary differential equations," in preceedings of the American Mathematical Society, 1994, pp. 743-748.

[15] L. Erbe, S. Hu, and H. Wang, "Multiple positive solutions of some boundary value problems," Mathematical Analysis and Applications, vol. 184, pp. 743-748, 1994.

[16] Y. Naito and S. Tanaka, "On the existence of multiple solutions of the boundary value problem for nonlinear second-order differential equations," Nonlinear Analysis, vol. 56, no. 4, pp. 919-935, 2004.

[17] R. Ma and B. Thompson, "Multiplicity results for second-order twopoint boundary value problems with nonlinearities across several eigenvalues," Applied Mathematics Letters, vol. 18, no. 5, pp. 587$595,2005$.

[18] J. Marcos do Ó, S. Lorca, and P. Ubilla, "Local superlinearity for elliptic systems involving parameters," Journal of Differential Equations, vol. 211, no. 1, pp. 1-19, 2005.

[19] J. Stoer and R. Bulirsch, Introduction to Numerical Analysis. New York: Springer-Verlag, 1980.

[20] J. Kevorkian, Perturbation Methods in Applied Mathematics. New York: Springer-Verlag, 1981.

[21] R. Carlson, A Concrete Introduction to Real Analysis. Boca Raton: CRC, 2006. 